\title{
Promoting Sustainable Print Behaviour
}

\author{
Jutta Willamowski Yves Hoppenot M. Antonietta Grasso \\ Xerox Research Centre Europe \\ 6, chemin de Maupertuis, 38240 Meylan, France \\ \{name.surname\}@xrce.xerox.com
}

\begin{abstract}
We have designed and developed the Personal Assessment Tool (PAT) to make users aware of the impact of their behavior on the environment and helping them to reduce it. PAT aims at promoting more sustainable print behavior in a corporate work environment. We have deployed and experimented PAT in our research center and describe this experiment along with preliminary findings and observations. From these findings we conclude that PAT definitely motivated the participating users to improve their print behavior. Nevertheless it also highlighted the constrained aspects of printing in a work environment calling for organizational changes of established work processes.
\end{abstract}

Keywords: These are the keywords. These are the keywords. These are the keywords.

\section{INTRODUCTION}

To face global warming issues and in general to promote sustainable development, many tools have been developed that help people to assess the impact of their behavior on the environment. These tools are often referred to as $\mathrm{CO} 2$ calculators. They collect input about user habits and behaviors, either dynamically in the background or as explicit input from the user, and provide as output a measure of how much they affect the environment, translating these behaviors into units like $\mathrm{CO} 2$ and energy consumption levels. Such tools are becoming widespread and their overall aim is to provide awareness, educate the user, and ultimately change this behavior in order to reduce his impact on the environment. Despite their simple mechanism, these tools are, however, very challenging to design in such a way to provide incentive to change habits and use of resources.

Froehlich et al. [2010] discuss the design of ecofeedback technology and various factors motivating pro-environmental behavior, namely information, goal-setting, comparison, commitment, rewards / penalties, and feedback.

In the light of these studies we have designed the Personal Assessment Tool (PAT) to make users aware of their impact on the environment and to help them to reduce it [Grasso et al. 2010]. PAT aims at promoting more sustainable print behavior in a corporate work environment. An essential component is the permanent awareness and feedback provided to the user. To support green transportation habits, Froehlich et al. [2009] choose a mobile phone display as appropriate way to provide permanent feedback to the user. To address print behavior, in PAT we choose a widget sitting permanently on the user's screen. In November 2011 we have deployed PAT in our research center. In the next section we quickly introduce PAT and then present this experiment along with preliminary findings and observations. From these findings we conclude that users were overall motivated to improve their print behavior. Nevertheless the work context imposes constraints that make it sometimes difficult to achieve a substantial change.

\section{THE PERSONAL ASSESSMENT TOOL EXPERIMENT}

The Personal Assessment Tool (PAT) aims at making users aware of their impact on the environment and at helping them to reduce it. It intercepts user actions in the background, analyzes them and provides its users with feedback about their behavior, its evolution over time and how it compares to that of their colleagues.

\subsection{How it works}

When users register to PAT they first go through a self-assessment step with questions about their printing habits making them reflect about their behaviour. Their self-estimate behavior is then confronted with their real, observed behaviour. This 
often results in an initial Aha-effect motivating the users to improve.

PAT then comes as a widget permanently sitting on the user's desktop. It displays a flower losing its petals over time thus materializing the user's impact on the environment (Figure 1).

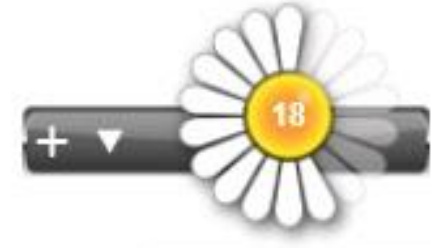

Figure 1: PAT widget, permanently sitting on the user's desktop.

To determine the user's impact on the environment PAT computes a cost for each print action in terms of a virtual currency called Green Points (GPs). Based on the user's observed past behavior, at the beginning of each month, PAT allocates to the user a personalized GPs quota. With each print action the user then consumes some of these GPs

Following a user's print action the flower widget is updated close to real time and provides thus permanent high-level and up-to-date feedback to the user. To further help users understand and improve their behavior, the PAT widget can be expanded to examine the users' print history and individual print actions in more detail (Figure 2).

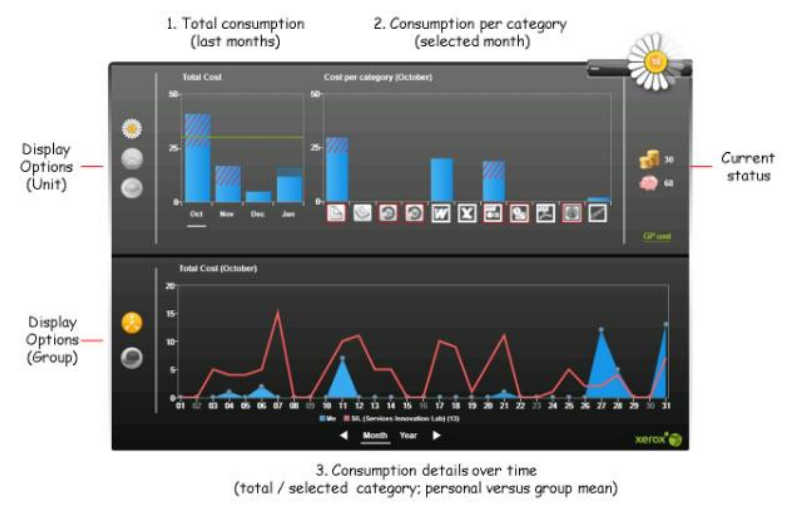

Figure 2: Expanded PAT widget.

\subsection{The experiment}

We have deployed PAT in our research center in November 2011 starting with a short center-wide introductory presentation and providing support whenever asked for. Over 50 people, i.e. more than half of the eligible employees registered within the first month on a voluntary basis. They were distributed over the different labs composing the research center on the one hand and the various work roles (researcher, developer, manager, assistant etc.) on the other hand.

During the trial period, we collected feedback on various occasions either spontaneously at any time or when assisting a user with the installation process. First of all, the self-assessment step proved to be effective. Indeed, several users reported that they had no idea of their printing consumption and habits and had difficulties estimating those. The self-assessment result confronting them with their past observed print behavior incited those users to immediately open the digest view to have a deeper look at their behavior. Several users (re-)discovered some particular (huge) print jobs they had recently issued and forgotten. Some of these print jobs concerned furthermore personal documents which is not a problem in this experiment setting, where it is accepted that work and personal life are often interleaved, still it helps to make people aware of the their personal usage of common (work) resources.

Having registered, right after the self-assessment, several users spontaneously shared and commented their personal quota through the PAT web site. This created somehow a sense of common objectives that could also be leveraged for other organizational activities and goals.

With the PAT flower widget sitting permanently on their desktop a number of users reported they felt it put a strong pressure on them inciting them to think twice before issuing any print job. One user even forwarded this pressure to the person replacing him during holidays by putting a post it on his screen urging to "print only what is really required". Some others reported that they sometimes wanted to close or uninstall the widget in order to get rid of this pressure particularly when they felt that the current (bad) status shown by the flower widget was not their fault, i.e. they had only printed what was required by their job but it had been an exceptionally high volume this time due to some unusual tasks.

Another person wanted to uninstall PAT, because her print consumption did not reflect her own working style but that of her boss traveling a lot and requiring her therefore to print lots of material to take away at such occasions. She was not only concerned about the way her personal behavior was impacted, but furthermore afraid that this would put bad light on her whole group when the results of the experiment would be presented to everyone. As discussed also by [Sellen 2003] it is indeed important to recognize that printing and working with paper documents is not always something the individual can fully control but rather depends a lot on the job requirements and context. Several users pointed out particular work processes in place in the center that are based on paper rather than on digital documents and which they have to follow which in turn makes them lose their personal GPs. Among these processes many are imposed by the organization and cannot be changed by the individual, sometimes also due to legal issues like intellectual property, accounting 
etc. People asked if these processes could be changed or the corresponding documents recognized as required by the organization (and not by them personally) such that the corresponding GPs would not be withdrawn from their personal account.

Some other work processes correspond rather to individual work habits and could be improved after reflection. One person for instance, responsible for a particular process, learned to digitally sign the forms she had to handle in order to get rid of printing intermediate paper documents just for signing purposes. Another person responsible for ordering supplies had previously archived a printed version of the order before sending it for signature. After reflection she decided that finally keeping the digital version only was enough.

To reduce their print consumption several users reported they moved towards more digital competency, reading on screen instead of paper, and scanning through a document before deciding if it is worth printing and which pages are relevant. One user even reported that she now furthermore digitally annotates the papers she is reading and actually likes this working style.

To reduce reprinting of huge documents one person proposed to share such documents once printed within her group. Such document sharing promotes good work practices and fosters communities at the work place. Some users reported that they did not reprint documents to get rid of minor formatting problems as they did before the experiment. For instance they did not reprint an Excel document where - in the print out - the last column had moved to the next page, due to a last minute change.

A part of the center's print consumption was also due to contact with external visitors and partners. In this context some PAT user's work consists of taking care of these visitors and providing them with documentation and promotional material. This is difficult to change because it involves not only the organization itself and its image but also the external partners and their expectations. To be able to reduce corresponding printing needs it will be necessary to relate the change in behavior (providing less printed material) convincingly to the general environmental benefit (instead for instance to corporate financial savings) such that external partners accept and adhere to this change (similar to towel reuse in hotels as discussed by Goldstein et al. [2009]).

PAT also brought issues to the surface where users reacted inappropriately (and may be thought existing software would solve the problem for them): indeed, when a printer blocked or broke down, users with a job in the corresponding queue often did not cancel that job but rather re-sent it to another printer. Nevertheless, when the initial printer got repaired, the queued jobs were printed and the corresponding GPs removed from the user's accounts. PAT made them aware of this problem and incited them to remember canceling jobs when appropriate.

Overall most users reported that they liked the tool and were interested in the environmental issue (which is not surprising as the participation in the experiment was on a voluntary basis and users who did not buy in did not participate from the beginning). Some participants reported an evolution of their attitude towards the tool over time: in the beginning they somehow over-reacted (trying to completely stop printing); then, over time, they changed and adopted a reasonable attitude, printing what is necessary, but trying to reduce and improve where possible. One user reported that a longer period of time is needed to learn various strategies for better printing (for instance to avoid having only one line of text on the last page of a document). She also would have liked additional functionality allowing her to set up personal objectives and follow their achievement.

Finally the experiment also highlighted some usability issues: sometimes users have their desktop filled with applications and corresponding windows. In these cases, the PAT widget is covered and not visible.

Beside the qualitative results presented above, we are currently analyzing the data collected before and throughout the experiment, in particular the user's print job logs on one hand (starting from May 2011, i.e. 6 months before the start of the experiment), and the PAT server access logs on the other hand providing information about the usage of the widget over time including the details about when people opened the digest view and which information they accessed.

The analysis of the PAT usage log provides us with information about if and how people use the PAT widget and its features and which information they essentially look for. A first analysis of these data shows that the number of users connected during work hours was rather stable over time which mainly indicates that users had the widget on their screens continuously (i.e. did not uninstall PAT). The access and usage of the digest view varies a lot. Indeed, while most users accessed the digest view on the day they registered, only a few consulted it regularly afterwards. We still need to analyze if there is a link between opening the digest view to access particular information and changing behavior.

With our experiment we primarily focused at a qualitative analysis that provided the results described above. Even if the quantitative analysis of the print volume in terms of number of sheets consumed before and after the experiment start date shows that the overall consumption has decreased, and that this decrease was more important for PAT users than for other employees, the current observations do not allow concluding with sufficient confidence that this reduction was 
achieved thanks to the PAT system. This is due to the limited number of employees in the center on one hand and to the limited observation period (well below one year) on the other hand.

\section{DISCUSSION}

In this paper we have presented PAT, a tool that makes people aware of their print behavior in a work context. We are currently conducting an experiment of this system in our research center. Overall the feedback collected from the experiment indicates that users got well engaged with the tool, i.e. that the way PAT implements the ecomotivational factors works. The way PAT presents information and provides feedback about his print behavior to its user first during the self-assessment and second on a high level through the flower and on a more detailed level through the expanded view seems to be useful.

The PAT users were overall motivated to improve their print behavior from the start, participation in the experiment being optional. The feedback collected shows that PAT helped its users to move towards better print behavior: indeed several people got aware of particular bad work processes they owned, found how to improve them and in consequence changed their behavior. Nevertheless as described in the previous section, the work environment often imposes constraints on individuals that make it difficult for them to achieve a substantial change. PAT incited these employees to pinpoint processes in the organization where changes can bring substantial benefit. In order to enable further improvement, the organization has to react and adapt its processes moving from paper to digital and ultimately to the paper-free office as described in AlIM [2012].

Making employees point out problematic processes is something that existing commercial tools aiming at optimizing printing in work environments will have more difficulty to achieve. Such tools usually target more the administrative side and provide end users only with punctual intervention rather than permanent awareness and feedback. [GreenPrint] for instance assists its user when submitting a print job first proposing to remove irrelevant content before printing and second advising on appropriate printers and print options. [Preo Printelligence] allows administrators to define rules to trigger "unobtrusive notifications" that will be shown to end users in particular situations e.g. to inform them about the cost of their last print job or to propose them to learn about particular features.

After all it is still an open question how users will react to a tool like PAT over a longer period of time. Will people get used to the tool and will the impact thus decrease? To maintain and increase motivation, we plan to introduce gaming mechanisms into the system: defining and proposing performance levels, challenges and competitions to users, allowing them to define and bet on (i.e. commit to) individual or collective improvement targets can contribute to keep them motivated over time. It will make people talk about the tool and their common objectives and also enable them to find and share strategies to reduce their consumption and to identify organizational processes that should be changed. Displaying the information about the global consumption in the shared printer area as proposed by Pousman et al. [2008] is another possibility to trigger discussions.

Finally, to come back to our experiment, this experiment had the particularity that users participated on a voluntary basis. It would be interesting to carry out further experiments in contexts where participation is non-voluntary.

\section{REFERENCES}

AlIM, The paper free office - dream or reality, (2012), http://www.aiim.org/Research/IndustryWatch/Paper-Free-Capture-2012.

Froehlich J., Dillahunt, T., Klasnja P., Mankoff J., Consolvo S., Harrison B., James Landay, J. A. (2009), UbiGreen: Investigating a Mobile Tool for Tracking and Supporting Green Transportation Habits, paper presented at ACM Conference on Human Factors in Computing Systems CHI2009, Boston, MA, USA, April 4 9 ,

Froehlich, J., Findlater L., Landay, J. (2010), The design of Eco-Feedback Technology. paper presented at the ACM Conference on Human Factors in Computing Systems (CHI 2010), Atlanta, USA, April, 10-15.

Goldstein, N. J., Cialdini, R. B., Griscevicius, V. A (2009), Room with a Viewpoint: Using Social Norms to Motivate Environmental Conservation in Hotels, Journal of Consumer Research, 35, August 2009.

Grasso, A., Willamowski, J., Ciriza, V., Hoppenot, Y. (2010), A System Providing Environmental Feedback to Users of Shared Printers for Providing Environmental Feedback, paper presented at the Ninth International Conference on Machine Learning and Applications (ICMLA 2010), Washington, USA, December 12-14.

GreenPrint http://www.printgreener.com/

Pousman, Z., Rouzati, H., Stasko, J. (2008), Imprint, a Community Visualization of Printer Data: Designing for Open-ended Engagement on Sustainability, paper presented at Computer Supported Cooperative Work Conference (CSCW 2008), San Diego, USA, November, 812.

Preo Printelligence:http://www.preosoftware.com/

Sellen, A., Harper R. H. R (2003), The Myth of the Paperless Office, MIT Press Cambridge, 245 pp. 
Promoting sustainable print behaviour

Jutta Willamowski, Yves Hoppenot, Antonietta Grasso 